\title{
Estimating predictors of severity of Group A Streptococcus infection in pregnancy
}

Lee Reicher ${ }^{1}$, Emmanuel Attali ${ }^{2}$, Omri Dominsky², Yonni Cohen ${ }^{3}$, Jalal Abu-Hanna ${ }^{2}$, Ariel Many $^{1}$, Yariv Yogev ${ }^{4}$, and Yuval Fouks ${ }^{1}$

${ }^{1}$ Affiliation not available

${ }^{2}$ Tel-Aviv Sourasky Medical Center, Tel-Aviv, Israel. Affiliated to the Sackler Faculty of Medicine, Tel-Aviv University, Tel-Aviv, Israel.

${ }^{3}$ Tel Aviv University Sackler Faculty of Medicine

${ }^{4}$ Tel Aviv Sourasky Medical Center

June 29, 2021

\begin{abstract}
Objective: To identify the clinical characteristics of pregnancy associated group A streptococcus (GAS) infection and predictors for intensive care unit (ICU) admission. Design: A retrospective cohort study of culture-proven pregnancy-related GAS infections. Setting and population: a tertiary university-affiliated hospital between 1/2008-7/2020. Methods: Review patient's electronic records of patients. Main outcome measures: Incidence of pregnancy associated GAS, proportion given prophylaxis and admission to ICU. Results: Of the 143,750 who delivered during the study period, $66(0.04 \%)$ were diagnosed as having a pregnancy associated GAS infection. Fifty-seven of them (86.3\%) presented postpartum, and nine (13.6\%) had septic abortions. The most common presenting signs and symptoms among puerperal GAS, were postpartum pyrexia (72\%), abdominal pain and/or tenderness (33\%), and tachycardia (>100 bpm, 22\%). Thirteen women (19.6\%) developed streptococcal toxic shock syndrome (STSS): 10 of them delivered vaginally, two had caesarean deliveries. Predictors for STSS and ICU admission were: antibiotic administration $>24$ hours from presentation postpartum, tachycardia, and a C-reactive protein level $>200 \mathrm{mg} / \mathrm{L}$. Women that received antibiotic prophylaxis during labour had a significantly lower rate of STSS (0 vs $10,22.7 \%$; $=0.04)$, as evidenced by the delayed interval from delivery to the first presentation of infection among those who received prophylaxis during labour $(8 \pm 4.8$ vs $4.8 \pm 4.2$ hours, $\mathrm{P}=0.008)$. Conclusion: Deferral of medical intervention $>24$ hours from the first registered abnormal sign had the most important impact on deterioration of women with invasive puerperal GAS. Antibiotic prophylaxis during labour in women with GAS may reduce complications
\end{abstract}

Estimating predictors of severity of Group AStreptococcus infection in pregnancy Lee Reicher, Emanuel Attali, Omri Dominski, Yoni Cohen, Abu-Hanna Jalal, Ariel Many, Yariv Yogev, Yuval Fouks

${ }^{a}$ Lis Maternity Hospital, Department of Obstetrics and Gynecology and bivision of Epidemiology, Tel Aviv Sourasky Medical Center, Tel Aviv, Israel, affiliated to the ${ }^{c}$ Sackler School of Medicine, Tel Aviv University, Tel Aviv, Israel.

Corresponding author: Yuval Fouks, MD, Lis Maternity Hospital, Department of Obstetrics and Gynecology, Sourasky Medical Center, 6 Weizmann Street, Tel Aviv, 6423906 Israel.

Telephone: +972-52-4260712; Email address: Fouksi@gmail.com

Shortened running title: The impact of antimicrobial prophylaxis in labor on Group A Streptococcus infection. 


\section{Word count: 2752}

\section{Abstract}

Objective: To identify the clinical characteristics of pregnancy associated group A streptococcus (GAS) infection and predictors for intensive care unit (ICU) admission.

Design: A retrospective cohort study of culture-proven pregnancy-related GAS infections.Setting and population: a tertiary university-affiliated hospital between 1/2008-7/2020. Methods: Review patient's electronic records of patients.Main outcome measures : Incidence and onset time of pregnancy associated GAS, proportion given prophylaxis and admission to Intensive care unit.

Results: Of the 143,750 who delivered during the study period, $66(0.04 \%)$ were diagnosed as having a pregnancy associated GAS infection. Fifty-seven of them (86.3\%) presented postpartum, and nine (13.6\%) had septic abortions. The most common presenting signs and symptoms among puerperal GAS, were postpartum pyrexia (72\%), abdominal pain and/or tenderness (33\%), and tachycardia (>100 bpm, 22\%). Thirteen women (19.6\%) developed streptococcal toxic shock syndrome (STSS): 10 of them delivered vaginally, two had caesarean deliveries. Predictors for STSS and ICU admission were: antibiotic administration $>24$ hours from presentation postpartum, tachycardia, and a C-reactive protein level $>200 \mathrm{mg} / \mathrm{L}$. Women that received antibiotic prophylaxis during labour had a significantly lower rate of STSS (0 vs $10,22.7 \% ; P=0.04)$, as evidenced by the delayed interval from delivery to the first presentation of infection among those who received prophylaxis during labour ( $8 \pm 4.8$ vs $4.8 \pm 4.2$ hours, $P=0.008$ ).

Conclusion: Deferral of medical intervention $>24$ hours from the first registered abnormal sign had the most important impact on deterioration of women with invasive puerperal GAS. Antibiotic prophylaxis during labour in women with GAS may reduce associated complications.

Keywords: Streptococcus pyogenes, group A Streptococcus, streptococcal infections, postpartum women.

'Tweetable abstract': The administration of antibiotic prophylaxis during labour for various indications is associated with significantly fewer occurrences of streptococcal toxic shock syndrome (STSS).

Predictors for intensive care unit admission were an empirical antibiotic administration longer than $24 \mathrm{~h}$ from presentation, postpartum tachycardia, and C-reactive protein $>200 \mathrm{mg} / \mathrm{L}$.

A seasonal distribution of group A Streptococcus (GAS) infection occurred between October and April.

We believe our results may aid in establishing a screening tool for carriers of group A streptococcal infection in pregnancy in order to prevent STSS.

\section{Introduction}

Gram-positive beta-haemolytic group A Streptococcus (GAS) bacteria, cause infections responsible for a wide range of diseases in humans. (1-3). GAS-associated endometritis is a potentially life-threatening event during pregnancy and postpartum $(2,4,5)$. GAS infections have re-emerged since the 1980s (6) after a period of continuous decline in infection rates associated with infection control measures and penicillin use. They now account for more than 75,000 yearly deaths worldwide (2). The attack rate of invasive GAS infection is 20 -fold higher for pregnant and postpartum women compared to non-pregnant women (6). Approximately 85-93\% of infections occur postpartum among patients with pregnancy-related GAS infection $(7,8)$. Risk factors for intrapartum and postpartum GAS infection include upper respiratory tract infection prior to delivery, contact with carriers of GAS infection during pregnancy (9), and risk factors attributed to the delivery itself, such as preterm premature rupture of membranes (PPROM) (1), mucosal damage, and emergency caesarean deliveries (10).

A rate of maternal morbidity and even mortality was reportedly as high as $60 \%$ when streptococcal toxic shock syndrome (STSS) develops (11). The severity of maternal infection in cases of pregnancy-associated GAS infection can be difficult to predict, and there is a lack of studies on predictions of pregnancy-associated GAS disease severity. It is plausible to assume that late recognition of pregnancy-associated GAS is related 
to substantial morbidities, such as STSS, intensive care (ICU) admissions, septic shock, surgical intervention, and emergency hysterectomy.

This study aimed to determine the risk factors associated with GAS disease severity, such as maternal STSS and rates of admission to the ICU. The specific objective was to assess the impact of a delay in recognition of the infection or pathogen subtype and the provision of appropriate treatment on maternal instability and clinical deterioration.

\section{Methods}

\section{Study design}

This was a retrospective cohort study performed in a single university-affiliated tertiary medical center with an annual birth rate of approximately 12,000 deliveries. Comprehensive electronic medical records were reviewed, and all cases of cultures positive for GAS that had been taken from various body sites and fluids between January 2008 and July 2021 were identified.

\section{Definition}

A GAS infection was defined by the isolation of the pathogen from a sterile liquid or tissue site. A postpartum GAS infection was defined as the isolation of GAS growth in association with a clinical infection during the postpartum period. Blood and urine cultures were routinely obtained from all patients with peripartum hyperpyrexia (fever $>38^{\circ} \mathrm{C}$ ). Additional cultures from the genital tract were obtained in cases of a suspected maternal infection. Each case of a positive culture was considered as being infectious since GAS is considered a specific pathogen and not a contaminant/coloniser.

\section{Data analysis}

The data were retrieved from electronic medical files and included demographics, medical history, risk factors for infection, recent self or family member illness, the absence of medical insurance and the presence of major clinical comorbidities, such as diabetes and an immunocompromised state. The presenting signs and symptoms included inflammatory markers upon admission [white blood cell (WBC) and C-reactive protein (CRP) levels] and positive results of bacteriologic cultures (from the cervix and blood). The obstetric history included the course of delivery, gestational age at delivery, screening for group B streptococci (GBS), duration of membrane rupture, and presence of intrapartum fever, defined as a single oral temperature measurement $>38^{\circ} \mathrm{C}$.

The bacterial identification tests from the clinical specimens were performed at the local microbiology laboratory using the Vitek MS and Vitek2 systems (bioMerieux, St. Louis, MO) according to the Clinical and Laboratory Standards Institute criteria. The characteristics of the GAS infections were also retrieved from medical files, including time to symptom onset, initial symptoms or signs of illness, maximal body temperature, laboratory workup, and time from first presentation of symptoms to antibiotic treatment. The complications, including adult respiratory distress syndrome, renal and hepatic failure, disseminated intravascular coagulation, transfer to the ICU, and mechanical ventilation, were also documented. Lastly, the data on the medical and surgical treatments (antibiotics, intravenous immunoglobulin administration, laparoscopy, and hysterectomy) were collected and reviewed.

\section{Epidemiologic investigations}

In accordance with the medical centre's policy, an epidemiologic investigation was carried out after every case of postpartum GAS infection, and it included screening for group A streptococci carriage from all healthcare workers who treated the patients from the time of admission. Personnel screening included cultures of the throat, rectum, and skin lesions (if present). An institutional investigation was carried out by The Infection Control Unit in cases of GAS infection-related isolation, and the healthcare workers who had been screened during the investigation were sent to the national streptococci reference laboratory for subtyping analysis in cases of hospital-acquired infection. 


\section{Antibiotic prophylaxis and treatment regimens}

The departmental antibiotic protocol included: antimicrobial prophylaxis (2 g cefazolin intravenously 30 min before skin incision) in cases of caesarean deliveries. If PPROM occurred prior to 34 weeks and there were no signs of chorioamnionitis or foetal distress, the pregnancies were managed expectantly until 35 weeks at which time they were routinely induced. Expectant management included a five-day antibiotic course in accordance with The Mercer protocol for PPROM. This consisted of intravenous treatment with $8 \mathrm{~g} / \mathrm{d}$ of ampicillin for $48 \mathrm{~h}$ and a single parenteral dose of $500 \mathrm{mg}$ of azithromycin followed by five days of parenteral treatment with $1.5 \mathrm{~g} / \mathrm{d}$ of amoxicillin. If a suspicion of chorioamnionitis arose, treatment with intravenous ampicillin $(8 \mathrm{~g} / \mathrm{d})$ and gentamycin $(5 \mathrm{mg} / \mathrm{kg} / \mathrm{d})$ was initiated. Penicillin-based antibiotics and intravenous clindamycin (900 tid) were administered after the diagnosis of a GAS-related illness had been established.

\section{Statistical analysis}

Descriptive statistics are expressed as median, mean, standard deviation, minimum and maximum values, and interquartile ranges (IQR). Student's $t$-test was used for parametric variables that were normally distributed, and a Welch's $t$-test or a Mann-Whitney $U$ test was used for non-parametric variables that were non-normally distributed. Fisher's exact test was applied for small samples of categorical variables. All tests were two-sided, and $p$-values of $<0.05$ were considered significant. Bootstrap resampling with random computer-generated iterations was applied in cases of $n=0$.

\section{Results}

Incidence During the study period, there were 143,750 deliveries, 66 women identified with group A streptococci-positive in pregnancy or post-partum. Of these 57 cases of puerperal GAS-, 42 (73.1\%) delivered vaginally, $5(8.7 \%)$ by instrumental vacuum extraction, and $10(26.3 \%)$ by caesarean delivery. Nine women $(13.6 \%)$ diagnosed with septic abortions and had undergone dilation and curettage during their first and early second trimesters. During the study period, there was an incidence of one puerperal GAS infection per 2521 deliveries (0.04\%) during the 12.5-year study period. Fifteen GAS-infected women were treated with antibiotics by routine protocols during labour: specifically, nine women during a caesarean delivery, three because of a GBS-positive test result, and three others due to a fever during labour. Most of the identified cases of infection occurred between October-April $(n=52,78.7 \%)$ compared to the period between May-September $(n=14,21.2 \%, P=0.0001)$ (Table 1$)$.

\section{Clinical presentation}

The most common presenting signs and symptoms of infection among puerperal GAS infection were postpartum fever (48 patients, $84.2 \%$ ), abdominal pain and/or tenderness $(22,38.5 \%)$, and/or tachycardia (15, $26.8 \%$ ). Less common symptoms were back pain, headache, weakness, and dyspnoea. Cultures positive for GAS infection were obtained from various body sites and sterile liquids, mostly from the female genital tract (uterus, cervix, vagina, lochia, and placenta, $66 \%$ ), followed by blood (36\%), urine (13\%), caesarean delivery and episiotomy wounds $(6 \%)$. There were 28 puerperal women $(49.1 \%)$ with GAS-positive cultures originating from more than one sites.

\section{Complications}

Complications and treatment of pregnancy associated GAS infections are presented in Table 2. Thirteen women $(19.6 \%)$ developed STSS, of whom 10 delivered vaginally and two by caesarean delivery. One woman underwent a septic abortion by undergoing dilation and evacuation of the uterine cavity. In the post-partum group, the infectious prodrome occurred soon after birth, and the STSS was diagnosed within 48 hours of delivery in eight of the 13 women (61\%), between 2 to 7 days in three, and more than one week postpartum in the remaining two. Positive genital tract cultures for GAS infection (from the uterus, cervix, vagina, lochia, or placenta) were obtained from 11 women $(84.6 \%)$. Blood cultures were positive in six women. Organ complications included disseminated intravascular coagulation $(n=4)$, acute respiratory distress syndrome $(n=8)$, acute liver failure $(n=4)$, acute renal injury $(n=2)$, and respiratory failure necessitating intubation $(n=4)$. 


\section{Intensive care stay}

The characteristics of the women with a pregnancy associated GAS, transferred to the ICU compared to women hospitalized in the maternity department are presented in Table 3 . The groups were similar with regard to age, body mass index, and mode of delivery. The rate of women who presented with tachycardia $(>100 \mathrm{bpm})$ was higher among the ICU group ( $50 \%$ vs $17.7 \%, P=0.02)$. The rate of women who showed abnormal signs/symptoms less than $48 \mathrm{~h}$ after delivery was higher among women admitted to the ICU (61\% vs $28 \%, P=0.02)$. The rate of women who were first started on antibiotic treatment more than 24 hours from presentation was also higher among those in the ICU group $(98 \%$ vs $38.4 \% P<0.001)$. Six of the women who had been transferred to the ICU also received intravenous immunoglobulin. Exploratory laparoscopy or laparotomy was performed in six cases in which the patient had failed to recover following antibiotics and supportive treatment in the ICU. The average length of hospitalisation in the ICU was 18 days (range 8-54 days). There were no cases of maternal deaths and no cases of documented neonatal GAS infection.

\section{Epidemiologic investigations}

Cases of septic abortion were not investigated because the symptoms had appeared before hospitalization and, as such, were assumed to be community-acquired. Regarding post-natal cases subtypes: identical emmtype GAS infection was obtained from throat swabs of all members of the hospital staff with direct patient contact in seven cases. The dominant emm strains that were isolated were: emm $14.3(n=3)$, emm $22(n=$ $3)$, emm $75(n=4)$, and emm $11(n=3)$ and all of the affected patients were admitted to the ICU (Table 4). Figure 1 represents the multi-year analysis of infections in the medical centre. Five infections were deemed to be acquired from the hospital staff, and three of them that occurred in March 2008 were identified as having the same nidus of infection. Therefore, the overall rate of a proven hospital personal associated GAS infection was $8.7 \%$.

\section{Prophylaxis in labor}

Table 4 describes the characteristics of the women who either received or not antibiotics during labor and subsequently developed puerperal GAS infection. Those who received antibiotic prophylaxis during labor for various reasons (primarily due to a positive GBS screen or a caesarean delivery) had significantly lower rates of a subsequent STSS [0 vs $10(22.7 \%), P=0.04$ ], although the time from delivery to septic presentation was significantly longer ( $8 \pm 4.8$ vs $4.8 \pm 4.2), P=0.008)$. Of note, other complications, such as admission to the ICU and need to undergo laparotomy/explorative laparoscopy and hysterectomy were more numerous in those cases but the values did not reach a level of statistical significance.

\section{Discussion}

\section{Main findings}

This study sought to report the clinical consequences of women diagnosed with pregnancy-associated GAS. Its main findings were:

1. Women who received antibiotic prophylaxis during labor for various reasons had less severe infectious characteristics, such as STSS. This observation was supported by a longer interval between delivery to the first infectious presentation in those who received antibiotics.

2. Predictors ICU admission were a tardive empirical antibiotic administration of more than $24 \mathrm{~h}$ after presentation postpartum and tachycardia.

3. In contrast to other reports on a $30 \%$ mortality rate, there were no incidences of maternal death among the women who had sustained SSTS in the current study.

4. Hospital staff transmission was relatively uncommon throughout the study period. There was a seasonal distribution of GAS infection in a multi-year analysis, with most cases having been identified between October and April.

In spite of the advances in medical care, pregnancy-associated GAS infection contributes significantly to maternal morbidity and mortality worldwide. Since the recognition of healthcare personnel-related puerperal 
sepsis by Semmelweis (13), extensive and costly efforts have been undertaken to investigate outbreaks of GAS infection from a common nosocomial source. Contrarily, the current study findings, taken together with previous data $(8,14)$, reveal that healthcare personnel test negative in most cases, and that infections are considered as being community acquired for the most part. The current study results are in accordance with earlier ones that reported a comparable rate of presumed staff-related transmission of GAS infection. The overall incidence of puerperal GAS infection in the current cohort was similar to that of a previous study from the same institution (14). Of all identified GAS infections in the current study, $86 \%$ occurred postpartum and $14 \%$ occurred antepartum and were followed by septic abortions, similar to previous (8).

\section{Strengths and limitations}

This study has several limitations that bear mention. First, its retrospective nature raises questions about the applicability and generalisation of the results to account for local strains and subtypes. Second, several variables were underpowered, such as the rates of hysterectomies and laparotomies, since the prophylactic antibiotic analysis used only relatively small numbers. Furthermore, it was not possible to identify specific risk factors (e.g., throat colonisation and carriers among family members), which might serve as predictors for a possible future screening system for GBS prophylaxis. Lastly, it is very challenging to study the associations between emm types and clinical manifestations due to the vast number of circulating emm types.

\section{Interpretation}

The present study emphasises the importance of vital signs and the early recognition of infection for preventing a rapid infectious sequala. The regression analysis of the characteristics and clinical course of the 13 women whose pregnancy had been complicated with STSS and who were admitted to the ICU revealed that tachycardia as the first presenting sign and delay in providing antibiotic treatment were independent risk factors for severe infection that led to STSS and hospitalisation in the ICU. It is noteworthy that a high level of suspicion of STSS is always warranted because no single risk factor, specific clinical sign, or laboratory point to this serious complication. Since vital signs have been demonstrated as being of the utmost clinical importance with regard to infection prediction in cases of delay, the implementation of simple wearable technologies with the capacity for continuous monitoring of vitals should be considered.

Although GAS is a highly virulent pathogen, it has almost no resistance toward chemo-prophylactic agents commonly administered in labour. It was interesting to trace the natural history of patients diagnosed with GAS who received antimicrobial prophylaxis regardless of whether the source of infection was or was not known. The impact of antimicrobial prophylaxis has been described extensively by many authors as prolonging the disease-free state/latency period \{Mercer, 1997, Antibiotic therapy for reduction of infant morbidity after preterm premature rupture of the membranes. A randomized controlled trial. National Institute of Child Health and Human Development Maternal-Fetal Medicine Units Network\}. The eventual reduction in disease morbidity would be logical in this case, but it has yet to be proven. A large number of these individuals in the current work had received antimicrobial prophylaxis prior to undergoing a caesarean delivery, which could be a potential confounder. However, as stated in the results, the rates of caesarean delivery-related GAS infection were similar to its relative proportion in vaginal deliveries over the entire study period at the authors' institution. Assuming that antimicrobial prophylaxis at labor may indeed reduce the risk of severe infection, and specifically STSS, the calculated number needed to treat one case of STSS by antimicrobial prophylaxis is 14,375 women. The seasonality of the incidence of GAS has been reported before (15) in a large epidemiologic survey from 1975-2012. Those authors reported a significant seasonal variation in the incidence of GAS infections. They noticed a mid- to late-winter increase from January to April in both incidence and severity of infection (the diagnoses of sepsis, STSS, and death) among children and adults (38\% vs $16 \%$, respectively, $P<0.01$ ). Puerperal sepsis was diagnosed in only eight of their study women, precluding the drawing of any specific inferences for that subgroup. The current study had similar results, with most of the identified cases of GAS infection having occurred between October and April, although there was no significant correlation between specific emm-type and infection severity or seasonality.

\section{Conclusion}


This study documented the incidence, clinical course, and outcomes of pregnancy-related GAS infection and sought to elucidate the factors associated with infection severity. Deferral of medical intervention of more than 24 hours from the first signs of infection had the most important impact upon maternal deterioration. Antibiotic prophylaxis during labour may reduce GAS complications, warranting further study to detect specific risk factors and to reassess GAS screening, antibiotic prophylaxis and prevention policies.

\section{Contribution to Authorship}

L.R- Conception \& Design of Study, Manuscript Preparation, E.A- Data Collection, OD- Data Collection, YC- Data Analysis \& Interpretation, Statistical Analysis AHJ- Epidemiologic consultant, preformed the epidemiologic investigations AM- Epidemiologic consultant, Data Analysis \& Interpretation, YY- Manuscript Preparation, supervision, YF- Conception \& Design of Study, Manuscript Preparation

Funding statement: The authors received no financial support for the research, authorship, and/or publication of this article.

\section{Acknowledgement: none}

Ethics committee approval: This study was approved by the institutional ethics review board, approval number 0284-08-TLV: July 2015.

\section{References}

1. Anteby EY, Yagel S, Hanoch J, Shapiro M, Moses AE. Puerperal and intrapartum group A streptococcal infection. Infect Dis Obstet Gynecol 1999;7(6):276-82.

2. O'Loughlin RE, Roberson A, Cieslak PR, Lynfield R, Gershman K, Craig A, et al. The epidemiology of invasive group A streptococcal infection and potential vaccine implications: United States, 2000-2004. Clin Infect Dis 2007;45(7):853-62.

3. Tanaka H, Katsuragi S, Hasegawa J, Tanaka K, Osato K, Nakata M, et al. The most common causative bacteria in maternal sepsis-related deaths in Japan were group A Streptococcus: A nationwide survey. J Infect Chemother 2019;25(1):41-4.

4. Schuitemaker N, van Roosmalen J, Dekker G, van Dongen P, van Geijn H, Gravenhorst JB. Increased maternal mortality in The Netherlands from group A streptococcal infections. Eur J Obstet Gynecol Reprod Biol 1998;76(1):61-4.

5. Khan KS, Wojdyla D, Say L, Gülmezoglu AM, Van Look PF. WHO analysis of causes of maternal death: a systematic review. Lancet 2006;367(9516):1066-74.

6. Gustafson LW, Blaakær J, Helmig RB. Group A streptococci infection. A systematic clinical review exemplified by cases from an obstetric department. Eur J Obstet Gynecol Reprod Biol 2017;215:33-40.

7. Hamilton SM, Stevens DL, Bryant AE. Pregnancy-related group a streptococcal infections: temporal relationships between bacterial acquisition, infection onset, clinical findings, and outcome. Clin Infect Dis 2013;57(6):870-6.

8. Rottenstreich A, Benenson S, Levin G, Kleinstern G, Moses AE, Amit S. Risk factors, clinical course and outcomes of pregnancy-related group A streptococcal infections: retrospective 13-year cohort study. Clin Microbiol Infect. 2019;25(2):251.e1-.e4.

9. Leonard A, Wright A, Saavedra-Campos M, Lamagni T, Cordery R, Nicholls M, et al. Severe group A streptococcal infections in mothers and their newborns in London and the South East, 2010-2016: assessment of risk and audit of public health management. BJOG 2019;126(1):44-53.

10. Tuuli MG, Liu L, Longman RE, Odibo AO, Macones GA, Cahill AG. Infectious morbidity is higher after second-stage compared with first-stage cesareans. Am J Obstet Gynecol 2014;211(4):410.e1-6. 
11. Rimawi BH, Soper DE, Eschenbach DA. Group A streptococcal infections in obstetrics and gynecology. Clin Obstet Gynecol 2012;55(4):864-74.

12. Mercer BM, Miodovnik M, Thurnau GR, Goldenberg RL, Das AF, Ramsey RD, et al. Antibiotic therapy for reduction of infant morbidity after preterm premature rupture of the membranes. A randomized controlled trial. National Institute of Child Health and Human Development Maternal-Fetal Medicine Units Network. JAMA 1997;278(12):989-95.

13. Atalić B. Uz 200. obljetnicu rođenja Ignaza Philippa Semmelweisa [200th birth anniversary of Ignatius Philipp Semmelweis]. Acta Med Hist Adriat. 2018 Jun;16(1):9-18. Croatian. PMID: 30198270.

14. Shinar S, Fouks Y, Amit S, Pauzner D, Tarabeia J, Schechner V, Many A. Clinical Characteristics of and Preventative Strategies for Peripartum Group A Streptococcal Infections. Obstet Gynecol. 2016 Feb;127(2):227-32. doi: 10.1097/AOG.0000000000001238. PMID: 26942347.

15. Olafsdottir LB, Erlendsdóttir H, Melo-Cristino J, Weinberger DM, Ramirez M, Kristinsson KG, Gottfredsson M. Invasive infections due to Streptococcus pyogenes: seasonal variation of severity and clinical characteristics, Iceland, 1975 to 2012. Euro Surveill. 2014 May 1;19(17):5-14. Erratum in: Euro Surveill. 2016;21(10). doi: 10.2807/1560-7917.ES.2016.21.10.30158. PMID: 24821122.

Table 1. Characteristics of group A Streptococcus infection in pregnancy and postpartum $(n=66)$

\begin{tabular}{ll}
\hline Variable & Women $n=66 \mathbf{~ ( \% )}$ \\
Detection of an infection & \\
Postpartum $(<72$ hours) & $26(39.4)$ \\
Late postpartum & $27(40.9)$ \\
Infected abortion & $9(13.6)$ \\
Wound infection & $4(6.1)$ \\
Age, y & 32.7 \\
GA at delivery, weeks & $39 \pm 2$ \\
Mode of delivery & \\
NVD & $42(63.6)$ \\
VE & $5(7.6)$ \\
CS & $10(15.2)$ \\
Abortion & $9(13.6)$ \\
Positive GBS screening & $4(7.0)$ \\
Antibiotic treatment during labour & $15(22.7)$ \\
Presenting sign/symptom & \\
Fever & $48(72)$ \\
Abdominal pain & $22(33)$ \\
Tachycardia & $15(22)$ \\
Culture site & \\
Uterus & $44(66.6)$ \\
Urine & $9(13.6)$ \\
Blood & $24(36.3)$ \\
Wound & $4(6.1)$ \\
Positive from 2 sites & $28(42.4)$ \\
Time from sign/symptom to antibiotic treatment & \\
$<24$ h & $57(86.3)$ \\
$>24$ h & $9(13.6)$ \\
Epidemiology & \\
Hospital-acquired & $7(0.09)$ \\
Community-acquired & $59(90.9)$ \\
Season &
\end{tabular}


Table 2. Complications and treatment for group A Streptococcus infections in women with streptococcal toxic shock syndrome admitted to an intensive care unit $(n=12)$

\begin{tabular}{ll}
\hline Variable & $n \mathbf{( \% )}$ \\
Complication & \\
ARDS & $8(66.6)$ \\
Acute liver failure & $5(41.6)$ \\
Acute kidney failure & $2(16.2)$ \\
DIC & $4(33.3)$ \\
Treatment & \\
Antibiotics only & $6(50)$ \\
Intubation & $4(33.3)$ \\
IVIG & $6(66.6)$ \\
Laparotomy/laparoscopy & $6(66.6)$ \\
Uterine curettage & $2(16.2)$ \\
Hysterectomy & $4(33.3)$ \\
\hline
\end{tabular}

Table 3. Characteristics of women admitted to an intensive care unit $(n=13)$

Variable

Timing of infection

Post-partum $(<72 \mathrm{~h})$

Late post-partum

Wound infection

Age (y)

BMI $>30$

GDM

PROM (hours)

Mode of delivery

NVD

CS

Positive GBS screening

Presenting sign/symptom

Fever

Abdominal pain

Tachycardia (>100bpm)

C-reactive protein $(\mathrm{CRP})(\mathrm{mg} / \mathrm{L})$

Time to sign/symptom

$<48$ hours

$2-7$ days

$>7$ days

Time from sign/symptom to antibiotics treatment

$<24$ hours
Table 3. Characteristics of women admi ICU $(n=12)$

$9(75)$

$3(25)$

0

$32.4 \pm 5.5$

$2(15.4)$

$2(16.6)$

5.93

$10(83.3)$

$2(16.6)$

0

$9(75)$

$3(25)$

$6(50)$

$261.0(97.0)$

8 (66.6)

$3(25)$

1 (8.3)

5 (41.6.4) 
Table 4. Characteristics of women who received antibiotics during labour

Admitted to ICU

Time from delivery to presentation

Laparotomy/explorative laparoscopy

Hysterectomy

Streptococcal toxic shock syndrome
Table 4. Characteristics of wom

Antibiotics during labour $(n=$ $1(6.6 \%)$

$8( \pm 4.8)$

0

0

FIGURE LEGENDSFigure 1 A multi-year description of GAS infection and the results of the epidemiologic investigation

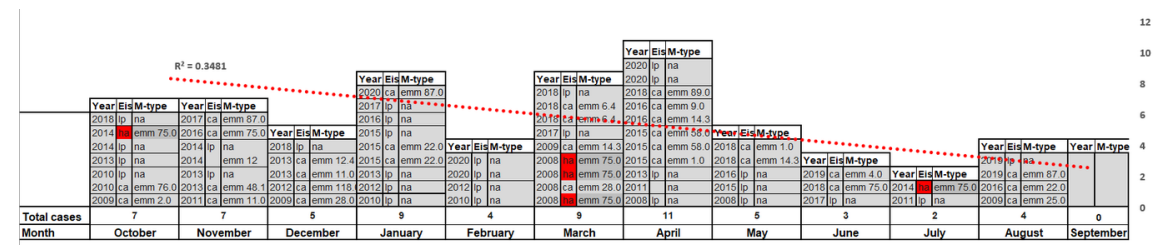

Eis: epidemiologic investigation status, Ca: community acquired, Ha: hospital-staff acquired, LpHa: low probability of hospital staff acquired

Flowchart

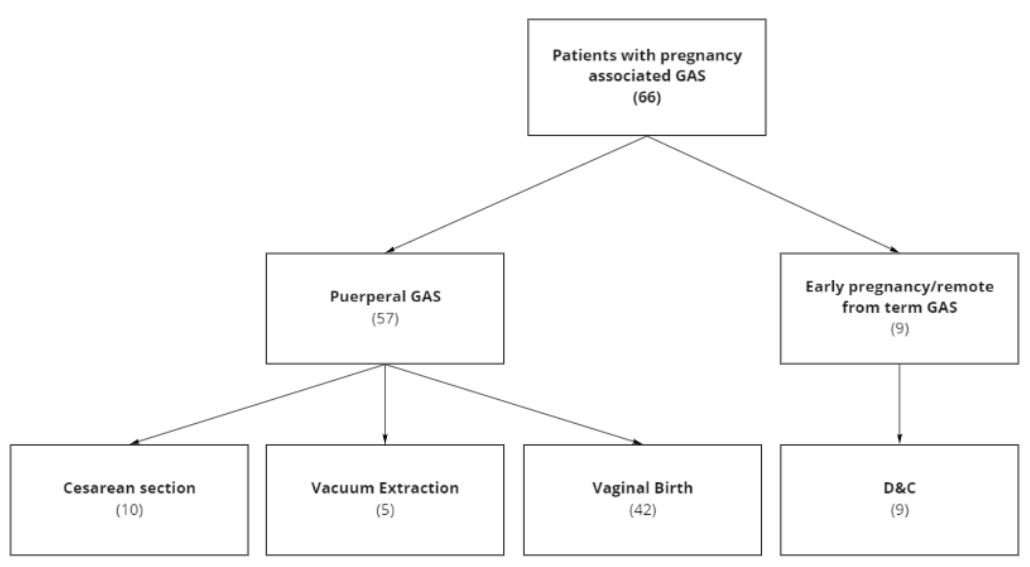

